Article

\title{
Extension of Elastic Models to Decagonal Quasicrystals
}

\author{
Wu Li * and Yiqing Shi
}

Institute of Science, Taiyuan University of Technology, Taiyuan 030024, China; baoxue0741@link.tyut.edu.cn

* Correspondence: liwu@tyut.edu.cn

Received: 15 March 2020; Accepted: 25 May 2020; Published: 1 June 2020

check for updates

\begin{abstract}
The main design of this paper is to adopt potential functions for solving plane defect problems originating from two-dimensional decagonal quasicrystals. First, we analyze the strict potential function theory for the plane problems of two-dimensional quasicrystals. To clarify effectiveness of the method, we give some examples and the results which can be precisely determined, including the elasticity and fracture theories of two-dimensional quasicrystals. These results maybe play a positive role in studying the fracture of two-dimensional quasicrystals in the future.
\end{abstract}

Keywords: quasicrystal; elasticity; plane problem; potential function theory

\section{Introduction}

A quasicrystal is seen as a new structure and was first observed by Shechtman et al. [1] and announced in 1984. As everyone knows, the physical basis of the elasticity of quasicrystals is considered to be the phenomenological theory of Landau and Lifshitz on the elementary excitation of condensed matters, in which two types of excitations, phonons and phasons, were considered for quasi-periodicity of materials [2]. The elastic properties of phonons and phasons of quasicrystals immediately led to widespread research, and theoretical and experimental solutions of a variety of defects and new physical thought play pivotal roles in the fracture mechanics of quasicrystalline materials [3-13]. In order to use fracture mechanics to solve practical problems, mastering the method of obtaining the solution of various defects in quasicrystalline materials is necessary. In the light of plane static problems of quasicrystals, the method of complex potential may be stringent and effective to obtain the analytic solutions. Of course, there are many numerical methods for obtaining the numerical solutions, for example, finite element method, finite difference method, etc.

In recent years, many solutions have been acquired and deduced for the plane cases of quaiscrytalline materials [14-19]. These research works clarify that the basic governing equation of plane problem of decagonal quasicrystals is the quadruple harmonic equation. So, the mathematical solution is much more complicated than those for conventional structural and foam materials. Mariano and Radi et al. developed the new methods to solve the crack or dislocation problem of icosahedral quasicrystals [20-22]. Li et al. deduced fundamental solutions with regard to one-dimensional hexagonal quasicrystals with some more complicated cracks, which are in thermo-elastic deformation [23,24]. Li et al. provided some solutions to two-dimensional quasicrystals by complex variable function method and finite difference methods [25,26]. A mass of models for the plastic deformation mechanism of quasicrystals was proposed by many researchers [27-33]. Moreover, other studies on plastic deformation of quasicrystals will not be listed here [34-36].

In this paper, the method of potential function theory is introduced for studying the plane problem of the quadruple harmonic equation. We will give some examples in following sections. 


\section{General Solution and Potential Function Theory}

A two-dimensional quasicrystal refers to a three-dimensional solid structure with the atom arrangement periodic along the $z$-direction and quasi-periodic on the $x y$ plane. Based on the plane elasticity theory of two-dimensional decagonal quasicrystals, it is clear that the deformation is limited in a plane perpendicular to the $\mathrm{z}$-axis. Then, the equations of deformation geometry in plane strain or plane stress state can be expressed by $[9,10,14]$ :

$$
\varepsilon_{i j}=\frac{1}{2}\left(\frac{\partial u_{i}}{\partial x_{j}}+\frac{\partial u_{j}}{\partial x_{i}}\right), w_{i j}=\frac{\partial w_{i}}{\partial x_{j}} .
$$

The displacement vectors are labeled as $u_{i}$ for phonon field and $w_{i}$ for phason field, respectively, which are both dependent on the spatial point $(x, y, z)=\left(x_{1}, x_{2}, x_{3}\right)$ in the real space. Similar to classical elasticity, these give rise to two displacement fields, so they are named elastic strain tensors $\varepsilon_{i j}$ and $w_{i j}$ and can be expressed by Equation (1).

Based on the stress tensors associated to strain tensors, the generalized Hooke's law for decagonal quasicrystals is [14]:

$$
\left\{\begin{array}{l}
\sigma_{x x}=L\left(\varepsilon_{x x}+\varepsilon_{y y}\right)+2 M \varepsilon_{x x}+R_{1}\left(w_{x x}+w_{y y}\right)+R_{2}\left(w_{x y}-w_{y x}\right), \\
\sigma_{y y}=L\left(\varepsilon_{x x}+\varepsilon_{y y}\right)+2 M \varepsilon_{y y}-R_{1}\left(w_{x x}+w_{y y}\right)-R_{2}\left(w_{x y}-w_{y x}\right), \\
\sigma_{x y}=\sigma_{y x}=2 M \varepsilon_{x y}+R_{1}\left(w_{y x}-w_{x y}\right)+R_{2}\left(w_{x x}+w_{y y}\right), \\
H_{x x}=K_{1} w_{x x}+K_{2} w_{y y}+R_{1}\left(\varepsilon_{x x}-\varepsilon_{y y}\right)+2 R_{2} \varepsilon_{x y}, \\
H_{y y}=K_{1} w_{y y}+K_{2} w_{x x}+R_{1}\left(\varepsilon_{x x}-\varepsilon_{y y}\right)+2 R_{2} \varepsilon_{x y}, \\
H_{x y}=K_{1} w_{x y}-K_{2} w_{y x}-2 R_{1} \varepsilon_{x y}+R_{2}\left(\varepsilon_{x x}-\varepsilon_{y y}\right), \\
H_{y x}=K_{1} w_{y x}-K_{2} w_{x y}+2 R_{1} \varepsilon_{x y}-R_{2}\left(\varepsilon_{x x}-\varepsilon_{y y}\right),
\end{array}\right.
$$

where $\sigma_{i j}$ are the phonon stresses and $H_{i j}$ are phason stresses and $C_{i j k l}, K_{i j k l}$ and $R_{i j k l}$ denote the phonon, phason and phonon-phason coupling elastic constants, and the elastic constants are $L=C_{12}$, $M=C_{66}=\left(C_{11}-C_{12}\right) / 2$, respectively.

If we neglect the body force, then the equilibrium equations can be expressed by:

$$
\frac{\partial \sigma_{i j}}{\partial x_{j}}=0, \frac{\partial H_{i j}}{\partial x_{j}}=0
$$

Based on the deformation compatibility equations,

$$
\frac{\partial^{2} \varepsilon_{x x}}{\partial y^{2}}+\frac{\partial^{2} \varepsilon_{y y}}{\partial x^{2}}=2 \frac{\partial^{2} \varepsilon_{x y}}{\partial x \partial y}, \frac{\partial w_{x x}}{\partial y}=\frac{\partial w_{x y}}{\partial x}, \frac{\partial w_{y y}}{\partial x}=\frac{\partial w_{y x}}{\partial y} .
$$

If we introduce three potential functions $\phi(x, y), \psi_{1}(x, y), \psi_{2}(x, y)$ as follows, the equilibrium (Equation (3)) is automatically satisfied,

$$
\left\{\begin{array}{l}
\sigma_{x x}=\frac{\partial^{2} \phi}{\partial y^{2}}, \sigma_{y y}=\frac{\partial^{2} \phi}{\partial x^{2}}, \sigma_{x y}=\sigma_{y x}=-\frac{\partial^{2} \phi}{\partial x \partial y^{\prime}}, \\
H_{x x}=\frac{\partial \psi_{1}}{\partial y}, H_{x y}=-\frac{\partial \psi_{1}}{\partial x}, H_{y x}=-\frac{\partial \psi_{2}}{\partial y}, H_{y y}=\frac{\partial \psi_{2}}{\partial x},
\end{array}\right.
$$

On the basis of the generalized Hooke's law (Equation (2)), we rewrite the equations by using all stress components to express all strain components. If we let

$$
\left\{\begin{array}{l}
\phi=c_{1} \nabla^{2} \nabla^{2} G \\
\psi_{1}=-\left(R_{1} \frac{\partial}{\partial y} \Pi_{1}+R_{2} \frac{\partial}{\partial x} \Pi_{2}\right) \nabla^{2} G \\
\psi_{2}=\left(R_{1} \frac{\partial}{\partial x} \Pi_{2}-R_{2} \frac{\partial}{\partial y} \Pi_{1}\right) \nabla^{2} G
\end{array}\right.
$$


in which

$$
\begin{aligned}
\nabla^{2} & =\frac{\partial^{2}}{\partial x^{2}}+\frac{\partial^{2}}{\partial y^{2}}, \Pi_{1}=3 \frac{\partial^{2}}{\partial x^{2}}-\frac{\partial^{2}}{\partial y^{2}}, \Pi_{2}=3 \frac{\partial^{2}}{\partial y^{2}}-\frac{\partial^{2}}{\partial x^{2}}, \\
c & =M\left(K_{1}+K_{2}\right)-2\left(R_{1}{ }^{2}+R_{2}{ }^{2}\right), c_{1}=\frac{c}{K_{1}-K_{2}}+M
\end{aligned}
$$

The set of these equations, after simple manipulations, can be reduced to a quadruple harmonic equation for the stress potential $G(x, y)$ as follows [14]:

$$
\nabla^{2} \nabla^{2} \nabla^{2} \nabla^{2} G=0
$$

in which

$$
\nabla^{2}=\partial^{2} / \partial x^{2}+\partial^{2} / \partial y^{2}
$$

It is clear that the general solution of Equation (7) is

$$
G=2 \operatorname{Re}\left(g_{1}(z)+\bar{z} g_{2}(z)+\frac{1}{2} \bar{z}^{2} g_{3}(z)+\frac{1}{6} \bar{z}^{3} g_{4}(z)\right)
$$

in which $g_{i}(z)(i=1,2,3,4)$ are analytic functions of a single complex variable $z=x+y i=r e^{i \theta}$.

Based on the fundamental solution (Equation (8)), by substituting Equation (8) into Equation (6), then substituting the resulting equations into Equation (5) we can obtain

$$
\left\{\begin{array}{l}
\sigma_{x x}=-32 c_{1} \operatorname{Re}\left(\Omega(z)-2 g_{4}^{\prime \prime \prime}(z)\right), \\
\sigma_{y y}=32 c_{1} \operatorname{Re}\left(\Omega(z)+2 g_{4}^{\prime \prime \prime}(z)\right) \\
\sigma_{x y}=\sigma_{y x}=32 c_{1} \operatorname{Im} \Omega(z) \\
H_{x x}=32 R_{1} \operatorname{Re}\left(\Theta^{\prime}(z)-\Omega(z)\right)-32 R_{2} \operatorname{Im}\left(\Theta^{\prime}(z)-\Omega(z)\right) \\
H_{x y}=-32 R_{1} \operatorname{Im}\left(\Theta^{\prime}(z)+\Omega(z)\right)-32 R_{2} \operatorname{Re}\left(\Theta^{\prime}(z)+\Omega(z)\right), \\
H_{y x}=-32 R_{1} \operatorname{Im}\left(\Theta^{\prime}(z)-\Omega(z)\right)-32 R_{2} \operatorname{Re}\left(\Theta^{\prime}(z)-\Omega(z)\right) \\
H_{y y}=-32 R_{1} \operatorname{Re}\left(\Theta^{\prime}(z)+\Omega(z)\right)+32 R_{2} \operatorname{Im}\left(\Theta^{\prime}(z)+\Omega(z)\right),
\end{array}\right.
$$

in which

$$
\Theta(z)=g_{2}{ }^{(I V)}(z)+\bar{z} g_{3}{ }^{(I V)}(z)+\frac{1}{2} \bar{z}^{2} g_{4}{ }^{(I V)}(z), \Omega(z)=g_{3}{ }^{(I V)}(z)+\bar{z} g_{4}{ }^{(I V)}(z)
$$

Meanwhile, the displacement field components can also be expressed by:

$$
\left\{\begin{array}{l}
u_{x}+i u_{y}=32\left(4 c_{1} c_{2}-c_{3}-c_{1} c_{4}\right) g_{4}^{\prime \prime}(z)-32\left(c_{1} c_{4}-c_{3}\right)\left(\overline{g_{3}^{\prime \prime \prime}(z)}+z \overline{g_{4}^{\prime \prime \prime}(z)}\right) \\
w_{x}+i w_{y}=\frac{32\left(R_{1}-i R_{2}\right)}{K_{1}-K_{2}} \overline{\Theta(z)}
\end{array}\right.
$$

in which

$$
c_{2}=\frac{(L+M)\left(K_{1}+K_{2}\right)+c}{4 c(L+M)}, c_{3}=\frac{\left(R_{1}^{2}+R_{2}^{2}\right)}{c}, c_{4}=\frac{\left(K_{1}+K_{2}\right)}{c} .
$$

By introducing the new functions for convenience such that

$$
g_{2}{ }^{(I V)}(z)=h_{2}(z), g_{3}{ }^{\prime \prime \prime}(z)=h_{3}(z), g_{4}^{\prime \prime}(z)=h_{4}(z)
$$

It is clear that all stress and displacement components can be expressed by $h_{i}(z)(i=2,3,4)$ based on the new four functions. When we use $h_{3}(z)+\gamma^{\prime}$ to replace the analytic function $h_{3}(z)$, and use 
$h_{4}(z)+\frac{32\left(c_{1} c_{4}-c_{3}\right)}{32\left(4 c_{1} c_{2}-c_{3}-c_{1} c_{4}\right)} \overline{\gamma^{\prime}}$ to replace the analytic function $h_{4}(z)$ [14], it will not change the stress and displacement field. So, we can get these complex functions written in the following form:

$$
\left\{\begin{array}{l}
h_{4}(z)=d_{1}(X+i Y) \ln z+B z+h_{4}{ }^{0}(z) \\
h_{3}(z)=d_{2}(X-i Y) \ln z+\left(B^{\prime}+i C^{\prime}\right) z+h_{3}{ }^{0}(z) \\
h_{2}(z)=\left(B^{\prime \prime}+i C^{\prime \prime}\right) z+h_{2}^{0}(z)
\end{array}\right.
$$

in which $B, B^{\prime}, C^{\prime}, B^{\prime \prime}$ and $C^{\prime \prime}$ are real constants, and $d_{1}, d_{2}, h_{4}{ }^{0}(z), h_{3}{ }^{0}(z)$ and $h_{2}{ }^{0}(z)$ can be marked as:

$$
\begin{aligned}
d_{1}= & \frac{1}{64 c_{1} \pi \times\left(32\left(4 c_{1} c_{2}-c_{3}-c_{1} c_{4}\right)+1\right)}, d_{2}=-\frac{4 c_{1} c_{2}-c_{3}-c_{1} c_{4}}{2 c_{1} \pi \times\left(32\left(4 c_{1} c_{2}-c_{3}-c_{1} c_{4}\right)+1\right)}, \\
& h_{4}^{0}(z)=\frac{a_{1}}{z}+\frac{a_{2}}{z}+\cdots \cdots, h_{3}^{0}(z)=\frac{b_{1}}{z}+\frac{b_{2}}{z}+\cdots \cdots, h_{2}^{0}(z)=\frac{\gamma_{1}}{z}+\frac{\gamma_{2}}{z}+\cdots \cdots,
\end{aligned}
$$

and

$$
B=\frac{1}{128 c_{1}}\left(\sigma_{1}+\sigma_{2}\right), B^{\prime}+i C^{\prime}=-\frac{1}{64 c_{1}}\left(\sigma_{1}-\sigma_{2}\right) e^{-2 i \alpha},
$$

in which $\sigma_{1}, \sigma_{2}$ represent principal stresses at infinity, and $\alpha$ denotes the angle of $\sigma_{1}$ and $x$-direction. If we take $z \rightarrow \infty$, it will obtain

$$
H_{y y}-H_{x x}+i\left(H_{x y}+H_{y x}\right)=-64\left(R_{1}+R_{2}\right) B^{\prime \prime}+i C^{\prime \prime}, B^{\prime \prime}+i C^{\prime \prime}=\frac{1}{64\left(R_{1}+R_{2}\right)}\left(\sigma_{1}{ }^{\prime}-\sigma_{2}{ }^{\prime}\right) e^{-2 i \alpha^{\prime}},
$$

where the generalized principal stresses at infinity are denoted as $\sigma_{1}{ }^{\prime}, \sigma_{2}{ }^{\prime}$, and $\alpha^{\prime}$ denotes the angle between $\sigma_{1}{ }^{\prime}$ and $x$-direction.

As we all know, in order to solve some complicated configurations of the elastic materials, Muskhelishvili [34] had adopted two kinds of rational conformal mapping. The first is constructed by

$$
z=\omega(\zeta)=R\left(\zeta+\sum_{k=0}^{n} d_{k} \zeta^{-k}\right) \sum_{k=0}^{n}\left|d_{k}\right| \leq 1
$$

This mapping can transform the exterior of the unit circle in the $\zeta$-plane into the exterior of the material with defects in the $z$-plane. We can obtain series expansion of the logarithmic item $\ln z=\ln \left[R \zeta\left(1+\sum_{k=0}^{n} d_{k} \zeta^{-(k+1)}\right)\right]=\ln R+\ln \zeta+\ln \left(1+\sum_{k=0}^{n} d_{k} \zeta^{-(k+1)}\right)$ on the basis of the transformation. Because of $|\zeta|>1$ outside the unit circle, it is clear to obtain $\sum_{k=0}^{n}\left|\frac{d_{k}}{\zeta^{k+1}}\right|<1$ and also get

$$
f(\zeta)=\ln \left(1+\sum_{k=0}^{n} d_{k} \zeta^{-(k+1)}\right)=\sum_{k=0}^{n} d_{k} \zeta^{-(k+1)}+\frac{1}{2}\left(\sum_{k=0}^{n} d_{k} \zeta^{-(k+1)}\right)^{2}+\cdots \cdots
$$

in which $f(\zeta)$ is analytic outside the unit circle. As a result of similar analysis, it is clear to obtain each item $h_{4}^{0}(z), h_{3}^{0}(z), h_{2}^{0}(z)$ of two dimensional decagonal quasicrystals, for example, the following formula for the series expansion,

$$
\frac{a_{1}}{z}=\frac{a_{1}}{R \zeta\left(1+\sum_{k=0}^{n} d_{k} \zeta^{-(k+1)}\right)}=\frac{a_{1}}{R \zeta}\left(1-\sum_{k=0}^{n} d_{k} \zeta^{-(k+1)}-\cdots \cdots\right)
$$

and so on. Based on these results, by inserting them into Equation (12), we have

$$
\left\{\begin{array}{l}
h_{4}(\zeta)=d_{1}(X+i Y) \ln \zeta+B \omega(\zeta)+h_{4}^{*}(\zeta) \\
h_{3}(\zeta)=d_{2}(X-i Y) \ln \zeta+\left(B^{\prime}+i C^{\prime}\right) \omega(\zeta)+h_{3}^{*}(\zeta) \\
h_{2}(\zeta)=\left(B^{\prime \prime}+i C^{\prime \prime}\right) \omega(\zeta)+h_{2}^{*}(\zeta)
\end{array}\right.
$$


in which

$$
h_{4}^{*}(\zeta)=\sum_{n=1}^{\infty} \alpha_{n} \zeta^{-n}, h_{3}{ }^{*}(\zeta)=\sum_{n=1}^{\infty} \beta_{n} \zeta^{-n}
$$

and:

$$
h_{2}^{*}(\zeta)=\sum_{n=1}^{\infty} \gamma_{n} \zeta^{-n}
$$

are single valued analytical functions of $|\zeta|>1$. The second reads

$$
z=\omega(\zeta)=R\left(\frac{1}{\zeta}+\sum_{k=0}^{n} C_{k} \zeta^{k}\right) \sum_{k=0}^{n}\left|C_{k}\right| \leq 1 .
$$

This mapping can transform the interior of the unit circle in the mapping plane into the exterior of the material with defects in the physical plane. In light of a similar analysis with the first kind mapping, we can obtain:

$$
\left\{\begin{array}{l}
\hat{h}_{4}(\zeta)=-d_{1}(X+i Y) \ln \zeta+B \omega(\zeta)+\hat{h}_{4}^{*}(\zeta) \\
\hat{h}_{3}(\zeta)=-d_{2}(X-i Y) \ln \zeta+\left(B^{\prime}+i C^{\prime}\right) \omega(\zeta)+\hat{h}_{3}^{*}(\zeta) \\
\hat{h}_{2}(\zeta)=\left(B^{\prime \prime}+i C^{\prime \prime}\right) \omega(\zeta)+\hat{h}_{2}^{*}(\zeta)
\end{array}\right.
$$

in which:

$$
\hat{h}_{4}^{*}(\zeta)=\sum_{n=1}^{\infty} \hat{\alpha}_{n} \zeta^{n}, \hat{h}_{3}^{*}(\zeta)=\sum_{n=1}^{\infty} \hat{\beta}_{n} \zeta^{n}, \hat{h}_{2}^{*}(\zeta)=\sum_{n=1}^{\infty} \hat{\gamma}_{n} \zeta^{n}
$$

are single valued analytical functions of $|\zeta|<1$.

The stress boundary conditions for plane elasticity of decagonal quasicrystals can be expressed by:

$$
\left\{\begin{array}{l}
\sigma_{x x} l+\sigma_{x y} m=T_{x}, \sigma_{y y} m+\sigma_{x y} l=T_{y}(x, y) \in L \\
H_{x x} l+H_{x y} m=h_{x}, H_{y y} m+H_{y x} l=h_{y}(x, y) \in L
\end{array}\right.
$$

where the point $(x, y) \in L$ denotes an arbitrary boundary point of a multi-connected quasicrystalline material, and meanwhile we need to consider:

$$
l=\cos (\mathbf{n}, x)=d y / d s, m=\cos (\mathbf{n}, y)=-d x / d s .
$$

Here $T=\left(T_{x}, T_{y}\right)$ and $h=\left(h_{x}, h_{y}\right)$ state the surface tractions and generalized surface tractions, and $\mathbf{n}$ shows the outer unit normal vector of an arbitrary boundary point, respectively.

Due to the Equation (9) and Equation (12) and the boundary conditions (Equation (15)), the boundary conditions on basis of our analytic functions can be written as:

$$
\left\{\begin{array}{l}
h_{4}(z)+\overline{h_{3}(z)}+z \overline{h_{4}{ }^{\prime}(z)}=\frac{i}{32 c_{1}} \int\left(T_{x}+i T_{y}\right) d s \quad z \in L \\
h_{4}{ }^{\prime \prime}(z)+\bar{z} h_{3}{ }^{\prime}(z)+\frac{1}{2} \bar{z}^{2} h_{4}{ }^{\prime \prime}(z)=\frac{i}{R_{2}-i R_{1}} \int\left(h_{x}+i h_{y}\right) d s \quad z \in L
\end{array}\right.
$$

\section{Applications}

\subsection{Problem and Analytical Solution of the Semi-Infinite Plane}

The physical problem considered in this paper is shown in Figure 1. The periodic is assumed to be orientated in the $z$-axis and the quasi-periodic plane is paralleled to the $x y$-plane. The problem 
we consider is the application of uniform compressive stress $q$ on an elastic semi-infinite plane of an isotropic body. In this case, the boundary conditions can be described by

$$
\left\{\begin{array}{l}
\left(\sigma_{y y}\right)_{y=0}=-q, \\
\left(\sigma_{x y}\right)_{y=0}=0, \\
H_{y y}=0, H_{y x}=0 .
\end{array}\right.
$$

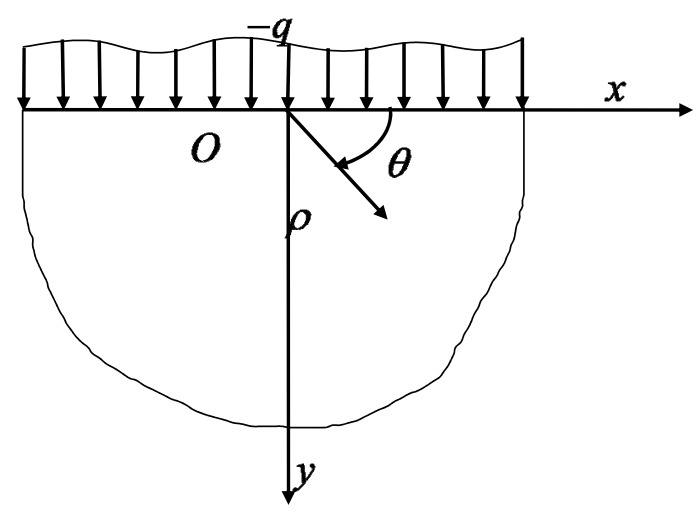

Figure 1. A schematic figure for semi-infinite plane of quasicrystals.

Performing the Fourier transform for Equation (7) and the boundary conditions, Equations (9) and (15), yield:

$$
\left(\frac{d^{2}}{d y^{2}}-\lambda^{2}\right)^{4} \hat{G}=0
$$

and

$$
\left\{\begin{array}{l}
D\left(-3 \lambda^{6} \frac{d \hat{G}}{d y}+2 \lambda^{4} \frac{d^{3} \hat{G}}{d y^{3}}+\lambda^{2} \frac{d^{5} \hat{G}}{d y^{5}}\right)=-Q(\lambda) \\
D\left(-3 \lambda^{5} \frac{d^{2} \hat{G}}{d y^{2}}+2 \lambda^{3} \frac{d^{4} \hat{G}}{d y^{4}}+\lambda \frac{d^{6} \hat{G}}{d y^{6}}\right)=0 \\
3 \lambda^{6} \frac{d \hat{G}}{d y}+10 \lambda^{4} \frac{d^{3} \hat{G}}{d y^{3}}+3 \lambda^{2} \frac{d^{5} \hat{G}}{d y^{5}}=0 \\
3 \lambda^{5} \frac{d^{2} \hat{G}}{d y^{2}}+10 \lambda^{3} \frac{d^{4} \hat{G}}{d y^{4}}+3 \lambda \frac{d^{6} \hat{G}}{d y^{6}}=0
\end{array}\right.
$$

in which

$$
\hat{G}(\lambda, y)=\frac{1}{\sqrt{2 \pi}} \int_{-\infty}^{+\infty} G(x, y) e^{-i \lambda x} d x
$$

In this case, the general solution of Equation (18) is

$$
\hat{G}=\left(A_{1}+A_{2}+A_{3}+A_{4}\right) e^{-\lambda y}+\left(B_{1}+B_{2}+B_{3}+B_{4}\right) e^{\lambda y}
$$

In order to ensure that the general solution is bounded when $y \rightarrow \infty$, it needs $B_{1}=B_{2}=B_{3}=$ $B_{4}=0$ and $\lambda$ has to take the absolute value (because $\lambda$ goes from $-\infty$ to $+\infty$ ). As a result, Equation (20) can be defined by:

$$
\hat{G}=\left(A_{1}+A_{2}+A_{3}+A_{4}\right) e^{-|\lambda| y}
$$

In addition, the boundary conditions (Equation (19)) are simplified to:

$$
\left\{\begin{array}{l}
\lambda^{2} A_{2}-4|\lambda| A_{3}+9 A_{4}=\frac{-Q(\lambda)}{8 D \lambda^{5}} \\
\lambda^{2} A_{2}-6|\lambda| A_{3}+21 A_{4}=0 \\
2 \lambda|\lambda| A_{1}-6 \lambda^{2} A_{2}+15|\lambda| A_{3}-30 A_{4}=0 \\
2 \lambda|\lambda| A_{1}-8 \lambda^{2} A_{2}+27|\lambda| A_{3}-75 A_{4}=0
\end{array}\right.
$$


The solutions of Equation (22) are:

$$
\left\{\begin{array}{l}
A_{1}=\frac{1}{2 \lambda \cdot|\lambda|}\left(\frac{77 Q(\lambda)}{48 D \lambda^{5}}+\frac{1295 Q(\lambda)}{24 D \lambda^{7}}\right) \\
A_{2}=\frac{-37 Q(\lambda)}{72 D \lambda^{7}} \\
A_{3}=\frac{11 Q(\lambda)}{144 D \lambda^{5} \cdot|\lambda|} \\
A_{4}=\frac{-Q(\lambda)}{108 D \lambda^{5}}
\end{array}\right.
$$

where $Q(\lambda)=\sqrt{2 \pi} q \delta(\lambda)$ is the Fourier transform of $q$.

From Equations (5), (6) and (8), it is easy to obtain the expressions of stress field:

$$
\begin{gathered}
\varphi=D\left(3 \cdot \frac{\partial^{5}}{\partial x^{4} \partial y}+2 \cdot \frac{\partial^{5}}{\partial x^{2} \partial y^{3}}-\frac{\partial^{5}}{\partial y^{5}}\right) G \\
\psi_{2}=(L+M)\left(K_{1}-K_{2}\right) R_{1} \cdot\left(-3 \cdot \frac{\partial^{6}}{\partial x^{5} \partial y}+10 \cdot \frac{\partial^{6}}{\partial x^{3} \partial y^{3}}-3 \cdot \frac{\partial^{6}}{\partial x \partial y^{5}}\right) G \\
\psi_{1}=-\frac{1}{R_{1}}\left(M K_{1}-R_{2}\right)\left[(L+2 M)\left(K_{1}-K_{2}\right)-2 R^{2}\right]\left(\frac{\partial^{6}}{\partial x^{6}}+3 \cdot \frac{\partial^{6}}{\partial x^{4} \partial y^{2}}+3 \cdot \frac{\partial^{6}}{\partial x^{2} \partial y^{4}}+\frac{\partial^{6}}{\partial y^{6}}\right) G \\
+(L+M)\left(K_{1}-K_{2}\right) R_{1} \cdot\left(-3 \cdot \frac{\partial^{6}}{\partial x^{5} \partial y}+10 \cdot \frac{\partial^{6}}{\partial x^{3} \partial y^{3}}-3 \cdot \frac{\partial^{6}}{\partial x \partial y^{5}}\right) G \\
\sigma_{x x}=\frac{\partial^{2} \varphi}{\partial y^{2}}=D \cdot\left(3 \cdot \frac{\partial^{7}}{\partial x^{4} \partial y^{3}}+2 \cdot \frac{\partial^{7}}{\partial x^{2} \partial y^{5}}-\frac{\partial^{7}}{\partial y^{7}}\right) G \\
\sigma_{y y}=\frac{\partial^{2} \varphi}{\partial x^{2}}=D \cdot\left(3 \cdot \frac{\partial^{7}}{\partial x^{6} \partial y}+2 \cdot \frac{\partial^{7}}{\partial x^{4} \partial y^{3}}-\frac{\partial^{7}}{\partial x^{2} \partial y^{5}}\right) G \\
\sigma_{x y}=\sigma_{y x}=-\frac{\partial^{2} \varphi}{\partial x \partial y}=-D \cdot\left(3 \cdot \frac{\partial^{7}}{\partial x^{5} \partial y^{2}}+2 \cdot \frac{\partial^{7}}{\partial x^{3} \partial y^{4}}-\frac{\partial^{7}}{\partial x \partial y^{6}}\right) G
\end{gathered}
$$

and the calculation process see Appendix A:

$$
\begin{aligned}
H_{x x}= & \frac{\partial \psi_{1}}{\partial y}=-\frac{1}{R_{1}}\left(M K_{1}-R_{2}\right)\left[(L+2 M)\left(K_{1}-K_{2}\right)-2 R_{1}^{2}\right]\left(\frac{\partial^{7}}{\partial x^{6} \partial y}+3 \cdot \frac{\partial^{7}}{\partial x^{7} \partial y^{3}}+3 \cdot \frac{\partial^{7}}{\partial x^{2} \partial y^{5}}+\frac{\partial^{7}}{\partial y^{7}}\right) \\
& +(L+M)\left(K_{1}-K_{2}\right) R_{1} \cdot\left(-3 \cdot \frac{\partial^{7}}{\partial x^{5} \partial y^{2}}+10 \cdot \frac{\partial^{7}}{\partial x^{3} \partial y^{4}}-3 \cdot \frac{\partial^{7}}{\partial x \partial y^{6}}\right) \\
H_{x y}= & -\frac{\partial \psi_{1}}{\partial x}=\frac{1}{R_{1}}\left(M K_{1}-R_{2}\right)\left[(L+2 M)\left(K_{1}-K_{2}\right)-2 R_{1}^{2}\right]\left(\frac{\partial^{7}}{\partial x^{7}}+3 \cdot \frac{\partial^{7}}{\partial x^{5} \partial y^{2}}+3 \cdot \frac{\partial^{7}}{\partial x^{3} \partial y^{4}}+\frac{\partial^{7}}{\partial x \partial y^{6}}\right) \\
& -(L+M)\left(K_{1}-K_{2}\right) R_{1} \cdot\left(-3 \cdot \frac{\partial^{7}}{\partial x^{6} \partial y}+10 \cdot \frac{\partial^{7}}{\partial x^{4} \partial y^{3}}-3 \cdot \frac{\partial^{7}}{\partial x^{2} \partial y^{5}}\right) \\
H_{y x}= & -\frac{\partial \psi_{2}}{\partial y}=(L+M)\left(K_{2}-K_{1}\right) R_{1} \cdot\left(-3 \cdot \frac{\partial^{7}}{\partial x^{5} \partial y^{2}}+10 \cdot \frac{\partial^{7}}{\partial x^{3} \partial y^{4}}-3 \cdot \frac{\partial^{7}}{\partial x \partial y^{6}}\right) \\
H_{y y}= & \frac{\partial \psi_{2}}{\partial x}=(L+M)\left(K_{1}-K_{2}\right) R_{1} \cdot\left(-3 \cdot \frac{\partial^{7}}{\partial x^{6} \partial y}+10 \cdot \frac{\partial^{7}}{\partial x^{4} \partial y^{3}}-3 \cdot \frac{\partial^{7}}{\partial x^{2} \partial y^{5}}\right)
\end{aligned}
$$

\subsection{Solutions to Dugdale Hypothesis of a Semi-infinite Crack in a Decagonal Quasicrystal}

There is a semi-infinite crack embedded in a semi-infinite specimen of decagonal quasicrystals. The distance between the tip of the crack and the free boundary is $b$. Suppose the portion $y=0, b<x<a$ of the crack is subjected to an equilibrium pressure stress $p$. Meanwhile, we suppose a Dugdale cohesive force zone is in the crack tip, whose length is $R$ and in the zone a traction $\sigma_{y y}=\sigma_{Y}$ is subjected, in which $\sigma_{Y}$ denotes the yield limit of the materials, as shown in Figure 2. 


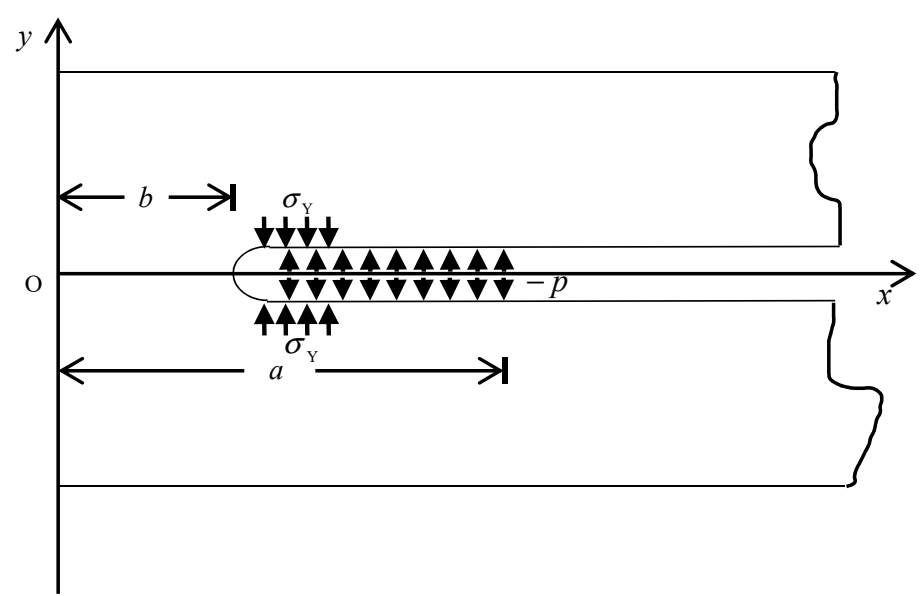

Figure 2. A schematic figure for a semi-infinite crack in a decagonal quasicrystal.

According to the superposition principle, the problem can be decoupled as the following two problems, in which the boundary conditions can be expressed as follows:

$$
\left\{\begin{array}{l}
\sigma_{x x}=\sigma_{x y}=0, H_{x x}=H_{x y}=0, x=0,-\infty<y<+\infty \\
\sigma_{y y}=-p, \sigma_{x y}=0, H_{y y}=H_{x y}=0, y=0, b<x<a \\
\sigma_{y y}=0, \sigma_{x y}=0, H_{y y}=H_{x y}=0, y=0, x>a \\
\sigma_{x x}=\sigma_{y y}=\sigma_{x y}=0, H_{x x}=H_{y y}=H_{x y}=0, \sqrt{x^{2}+y^{2}} \rightarrow+\infty .
\end{array}\right.
$$

and

$$
\left\{\begin{array}{l}
\sigma_{x x}=\sigma_{x y}=0, H_{x x}=H_{x y}=0, \quad x=0,-\infty<y<+\infty \\
\sigma_{y y}=\sigma_{Y}, \sigma_{x y}=0, H_{y y}=H_{x y}=0, y=0, b<x<b+R \\
\sigma_{y y}=0, \sigma_{x y}=0, H_{y y}=H_{x y}=0, \quad y=0, x>b+R \\
\sigma_{x x}=\sigma_{y y}=\sigma_{x y}=0, H_{x x}=H_{y y}=H_{x y}=0, \sqrt{x^{2}+y^{2}} \rightarrow+\infty .
\end{array}\right.
$$

3.2.1. Exact Solutions for Dugdale Hypothesis of a Semi-infinite Crack Based on Conformal Mapping

The conformal transformation has a form for this problem:

$$
z=\omega(\zeta)=b \sqrt{\frac{1}{2}-\frac{1}{4}\left(\frac{1}{\zeta}+\zeta\right)}
$$

It can transform the upper half $\zeta$ plane in the mapping plane into the region of the physical plane. Under the transformation (Equation (27)), the crack tip $z=b$ is mapped to $\zeta=-1$, while the points $z=\left(a, 0^{ \pm}\right)$and $z=\left(b+R, 0^{ \pm}\right)$are mapped to $\zeta=\sigma_{1,2}$ and $\zeta=\sigma_{1,2}^{\prime}$, i.e.,

$$
\sigma_{1}=1-\frac{2 a^{2}}{b^{2}}+\sqrt{\left(1-\frac{2 a^{2}}{b^{2}}\right)^{2}-1}, \sigma_{2}=\frac{1}{1-\frac{2 a^{2}}{b^{2}}+\sqrt{\left(1-\frac{2 a^{2}}{b^{2}}\right)^{2}-1}}
$$

and

$$
\begin{aligned}
\sigma^{\prime} & =1-\frac{2(b+R)^{2}}{b^{2}}+\sqrt{\left(1-\frac{2(b+R)^{2}}{b^{2}}\right)^{2}-1} \\
\sigma^{\prime} & =\frac{1}{1-\frac{2(b+R)^{2}}{b^{2}}+\sqrt{\left(1-\frac{2(b+R)^{2}}{b^{2}}\right)^{2}-1}}
\end{aligned}
$$


For the plane problem of the materials, we have the following functional equations from the boundary conditions (Equation (16))

$$
\left.\begin{array}{l}
h_{4}(\zeta)+\overline{h_{3}(-1)}+\frac{1}{2 \pi i} \int_{\gamma} \frac{\omega(\sigma)}{\overline{\omega^{\prime}(\sigma)}} \frac{\overline{h_{4}^{\prime}(\sigma)}}{\sigma-\zeta} d \sigma=\frac{1}{2 \pi i} \int_{L} \frac{f_{0}}{\sigma-\zeta} d \sigma \\
h_{3}(\zeta)+\overline{h_{4}(-1)}+\frac{1}{2 \pi i} \int_{\gamma} \frac{\overline{\omega(\sigma)}}{\frac{h^{\prime}(\sigma)}{h_{4}^{\prime}(\sigma)}} d \sigma=\frac{1}{2 \pi i} \int_{L} \frac{\overline{f_{0}}}{\sigma-\zeta} d \sigma
\end{array}\right\}
$$

in which

$$
f_{0}=\left\{\begin{array}{cl}
-p \omega(\sigma), & b<x<a \\
0, & \text { else }
\end{array}\right.
$$

for Equation (26a) and

$$
f_{0}=\left\{\begin{array}{c}
\sigma_{Y} \omega(\sigma), b<x<b+R \\
0, \text { else }
\end{array}\right.
$$

for Equation (26b).

In order to solve the stress intensity factor, it needs to have the function $h_{4}{ }^{\prime}(\zeta)$. We only give the results, ignoring the similar analysis and solving process with the above example:

$$
\begin{aligned}
h_{4}{ }^{\prime}(\zeta) & =\frac{1}{32 c_{1}} \cdot \frac{1}{2 \pi i} \cdot \int_{\sigma_{2}}^{\sigma_{1}} \frac{f_{0}}{(\sigma-\zeta)^{2}} d \sigma \\
& =\left.\frac{1}{32 c_{1}} \cdot \frac{p b}{\sqrt{2} \pi i} \cdot\left[\left(\frac{1+\zeta}{2 \sqrt{-2 \zeta}}\right) \ln \left|\frac{\sqrt{-2 \sigma}-\sqrt{-2 \zeta}}{\sqrt{-2 \sigma}+\sqrt{-2 \zeta}}\right|+\left(\frac{1-\zeta}{\sqrt{-2 \zeta}}\right)\left(\frac{1}{-2 \sigma+2 \zeta}\right)\right]\right|_{\sigma_{2}} ^{\sigma_{1}}
\end{aligned}
$$

for Equation (26a), and

$$
\begin{aligned}
h_{4}{ }^{\prime}(\zeta) & =\frac{1}{32 c_{1}} \cdot \frac{1}{2 \pi i} \cdot \int_{\sigma^{\prime}{ }_{2}}^{\sigma^{\prime}{ }^{\prime}} \frac{f_{0}}{(\sigma-\zeta)^{2}} d \sigma \\
& =-\left.\frac{1}{32 c_{1}} \cdot \frac{\sigma \mathrm{Y} b}{\sqrt{2} \pi i} \cdot\left[\left(\frac{1+\zeta}{2 \sqrt{-2 \zeta}}\right) \ln \left|\frac{\sqrt{-2 \sigma}-\sqrt{-2 \zeta}}{\sqrt{-2 \sigma}+\sqrt{-2 \zeta}}\right|+\left(\frac{1-\zeta}{\sqrt{-2 \zeta}}\right)\left(\frac{1}{-2 \sigma+2 \zeta}\right)\right]\right|_{\sigma_{2}^{\prime}} ^{\sigma_{1}^{\prime}}
\end{aligned}
$$

for Equation (26b).

Meanwhile, on the basis of the complex definition of stress intensity factor,

$$
K=K_{I}-i K_{I I}=\frac{\sqrt{2 \pi}}{16 c_{1}} \lim _{\zeta \rightarrow-1}\left\{\sqrt{\omega(\zeta)-\omega(-1)} \frac{h_{4}{ }^{\prime}(\zeta)}{\omega^{\prime}(\zeta)}\right\}=\frac{\sqrt{\pi}}{16 c_{1}} \frac{h_{4}{ }^{\prime}(-1)}{\sqrt{\omega^{\prime \prime}(-1)}}
$$

We substitute Equations (27) and (30) into Equation (31), and it yields:

$$
\begin{gathered}
K_{I}^{p}=\frac{4 p b}{\sqrt{3 \pi}} \cdot \frac{\sqrt{-2\left(1-\frac{2 a^{2}}{b^{2}}+\sqrt{\left(1-\frac{2 a^{2}}{b^{2}}\right)^{2}-1}\right)}}{2-\frac{2 a^{2}}{b^{2}}+\sqrt{\left(1-\frac{2 a^{2}}{b^{2}}\right)^{2}-1}} \\
K_{I}^{\sigma_{Y}}=-\frac{4 \sigma_{Y} b}{\sqrt{3 \pi}} \cdot \frac{\sqrt{-2\left(1-\frac{2(b+R)^{2}}{b^{2}}+\sqrt{\left(1-\frac{2(b+R)^{2}}{b^{2}}\right)^{2}-1}\right)}}{2-\frac{2(b+R)^{2}}{b^{2}}+\sqrt{\left(1-\frac{2(b+R)^{2}}{b^{2}}\right)^{2}-1}}
\end{gathered}
$$


The fact of the stresses at the crack tip must be finite, i.e., it needs total stress intensity factors equal to zero. Then it will result in the following equality that can determine the size of the cohesive force zone

$$
\frac{\sqrt{\left(1-\frac{2(b+R)^{2}}{b^{2}}+\sqrt{\left(1-\frac{2(b+R)^{2}}{b^{2}}\right)^{2}-1}\right)}}{2-\frac{2(b+R)^{2}}{b^{2}}+\sqrt{\left(1-\frac{2(b+R)^{2}}{b^{2}}\right)^{2}-1}}=\frac{p}{\sigma_{Y}} \cdot \frac{\sqrt{\left(1-\frac{2 a^{2}}{b^{2}}+\sqrt{\left(1-\frac{2 a^{2}}{b^{2}}\right)^{2}-1}\right)}}{2-\frac{2 a^{2}}{b^{2}}+\sqrt{\left(1-\frac{2 a^{2}}{b^{2}}\right)^{2}-1}}
$$

3.2.2. Exact Solutions for Dugdale Hypothesis of a Semi-Infinite Crack Based on Conformal Mapping

The conformal transformation has a form for this problem:

$$
z=\omega(\zeta)=\frac{b}{\sqrt{1-\zeta^{2}}}
$$

It also can transform the upper half $\zeta$ plane in the mapping plane into the region of the physical plane. Under the transformation (Equation (34)), crack tip $z=b$ is mapped to $\zeta=0$, while points $z=\left(a, 0^{ \pm}\right)$and $z=\left(b+R, 0^{ \pm}\right)$are mapped to

$$
\sigma_{1}=-\sqrt{1-\left(\frac{b}{a}\right)^{2}}, \sigma_{2}=+\sqrt{1-\left(\frac{b}{a}\right)^{2}} \text { and } \sigma_{1}^{\prime}=-\sqrt{1-\left(\frac{b}{b+R}\right)^{2}}, \sigma_{2}^{\prime}=+\sqrt{1-\left(\frac{b}{b+R}\right)^{2}} \text {. }
$$

On the similarity of the previous section, for the plane problem of the materials, we have the following functional equations from the boundary conditions (Equation (16)):

$$
\begin{aligned}
& h_{4}(\zeta)+\overline{h_{3}(0)}+\frac{1}{2 \pi i} \int_{\gamma} \frac{\omega(\sigma)}{\overline{\frac{\omega^{\prime}(\sigma)}{\omega(\sigma)}} \frac{\overline{h^{\prime}(\sigma)}}{\sigma-\zeta}} d \sigma=\frac{1}{2 \pi i} \int_{L} \frac{f_{0}}{\sigma-\zeta} d \sigma \\
& \left.h_{3}(\zeta)+\overline{h_{4}(0)}+\frac{1}{2 \pi i} \int_{\gamma} \frac{\overline{\omega(\sigma)}}{\omega^{\prime}(\sigma)} \frac{h^{\prime}(\sigma)}{\sigma-\zeta} d \sigma=\frac{1}{2 \pi i} \int_{L} \frac{\overline{f_{0}}}{\sigma-\zeta} d \sigma\right\} \text {, }
\end{aligned}
$$

in which

$$
f_{0}=\left\{\begin{array}{cl}
-p \omega(\sigma), & a<x<b \\
0, & \text { else }
\end{array}\right.
$$

for Equation (26a) and

$$
f_{0}=\left\{\begin{array}{c}
\sigma_{Y} \omega(\sigma), a<x<b+R \\
0, \quad \text { else }
\end{array}\right.
$$

for Equation (26b).

After the similar analysis and solving process with the above example, we only give the results of $h_{4}{ }^{\prime}(\zeta)$, i.e.,

$$
h_{4}{ }^{\prime}(\zeta)=\frac{1}{32 c_{1}} \cdot \frac{1}{2 \pi i} \cdot \int_{\sigma_{2}}^{\sigma_{1}} \frac{f_{0}}{(\sigma-\zeta)^{2}} d \sigma
$$

for Equation (26a) and

$$
h_{4}{ }^{\prime}(\zeta)=\frac{1}{32 c_{1}} \cdot \frac{1}{2 \pi i} \cdot \int_{\sigma^{\prime}}^{\sigma_{2}{ }_{1}} \frac{f_{0}}{(\sigma-\zeta)^{2}} d \sigma
$$

for Equation (26b).

If we let $\zeta=0$ in Equation (36), it will yield

$$
h_{4}^{\prime}(0)=-\left.\frac{p b}{32 c_{1}} \cdot \frac{1}{2 \pi i} \cdot \frac{\sqrt{1-\sigma^{2}}}{\sigma}\right|_{\sigma_{1}} ^{\sigma_{2}}
$$




$$
h_{4}{ }^{\prime}(0)=\left.\frac{\sigma_{Y} b}{32 c_{1}} \cdot \frac{1}{2 \pi i} \cdot \frac{\sqrt{1-\sigma^{2}}}{\sigma}\right|_{\sigma_{1}^{\prime}} ^{\sigma_{2}}
$$

Meanwhile, on the basis of the complex definition of stress intensity factor:

$$
K=K_{I}-i K_{I I}=\frac{\sqrt{2 \pi}}{16 c_{1}} \lim _{\zeta \rightarrow 0}\left\{\sqrt{\omega(\zeta)-\omega(0)} \frac{h_{4}{ }^{\prime}(\zeta)}{\omega^{\prime}(\zeta)}\right\}=\frac{\sqrt{\pi}}{16 c_{1}} \frac{h_{4}{ }^{\prime}(0)}{\sqrt{\omega^{\prime \prime}(0)}}
$$

We substitute Equations (34) and (37) into Equation (38) and it yields

$$
\begin{gathered}
K_{I}=\frac{4 p \sqrt{b}}{\sqrt{2 \pi}} \cdot \frac{b}{\sqrt{a^{2}-b^{2}}}, \\
K_{I}=-\frac{4 \sigma_{Y} \sqrt{b}}{\sqrt{2 \pi}} \cdot \frac{b}{\sqrt{(b+R)^{2}-b^{2}}}
\end{gathered}
$$

Based on the same approach of the preceding section, we can determine the size of cohesive force zone.

$$
R=\sqrt{\left(\frac{\sigma_{Y}}{p}\right)^{2} \cdot\left(a^{2}-b^{2}\right)+b^{2}}-b
$$

\section{Discussion and Conclusion}

A very important subject in the study of the mechanical behavior of decagonal quasicrystals are the defect problems. Of course, it is very difficult to treat the problems including notch and crack due to the complicated configuration. By introducing potential function theory, we analyze the strict theory for the plane problems of two-dimensional quasicrystals. As examples for applications, one is a semi-infinite plane in a decagonal quasicrystal, the other is the Dugdale hypothesis of a semi-infinite crack in a decagonal quasicrystal. Meanwhile, the obtained results in this paper are expressed in some exact analytical expressions, which maybe provide a useful theoretical base for the plane problems of decagonal quasicrystals. In the process, the successful application of potential function theory plays a leading role in solving these problems. We further obtain some important analytical solutions for decagonal quasicrystals. For example, the size of the cohesive force zone of the generalized cohesive force model of decagonal quasicrystals and the stress intensity factors (Equations (32) and (39)) are useful to the next works which are correlative to the study of the fracture mechanics of decagonal quasicrystals. Significantly, the two results of Equations (33) and (40) are approximately equal. These facts show that the potential function theory is very successful in solving such mechanical fracture problems.

Author Contributions: Conceptualization, W.L. and Y.S.; methodology, W.L.; formal analysis, W.L.; investigation, Y.S.; resources, Y.S.; writing-original draft preparation, W.L.; writing-review and editing, W.L.; project administration, W.L.; funding acquisition, W.L. All authors have read and agreed to the published version of the manuscript.

Funding: The work is supported by the National Natural Science Foundation of China (No. 11402158) and the Qualified Personnel Foundation of Taiyuan University of Technology (Grant No. tyut-rc201358a).

Conflicts of Interest: The authors declare no potential conflicts of interest with respect to the research, authorship and/or publication of this paper.

\section{Appendix A. The Calculation Process of Equation (25) of Section 3}

Performing the Fourier transform for the above results in the $x$ direction

$$
F\left(\sigma_{x x}\right)=D \cdot\left(-\frac{d^{7} \hat{G}}{d y^{7}}-2 \lambda^{2} \frac{d^{5} \hat{G}}{d y^{5}}+3 \lambda^{4} \frac{d^{3} \hat{G}}{d y^{3}}\right)
$$




$$
\begin{gathered}
F\left(\sigma_{x y}\right)=F\left(\sigma_{y x}\right)=D \cdot\left(-i \lambda \frac{d^{6} \hat{G}}{d y^{6}}+2 i \lambda^{3} \frac{d^{4} \hat{G}}{d y^{4}}-3 i \lambda^{5} \frac{d^{2} \hat{G}}{d y^{2}}\right) \\
F\left(\sigma_{y y}\right)=D \cdot\left(\lambda^{2} \frac{d^{5} \hat{G}}{d y^{5}}-2 \lambda^{4} \frac{d^{3} \hat{G}}{d y^{3}}-3 \lambda^{6} \frac{d \hat{G}}{d y}\right) \\
F\left(H_{x x}\right)=-\frac{1}{R_{1}}\left(M K_{1}-R_{2}\right)\left[(L+2 M)\left(K_{1}-K_{2}\right)-2 R_{1}^{2}\right]\left(-\lambda^{6} \frac{d \hat{G}}{d y}+3 \lambda^{4} \frac{d^{3} \hat{G}}{d y^{3}}-3 \lambda^{2} \frac{d^{5} \hat{G}}{d y^{5}}+\frac{d^{7} \hat{G}}{d y^{7}}\right) \\
+(L+M)\left(K_{1}-K_{2}\right) R_{1} \cdot i \cdot\left(3 \lambda^{5} \frac{d^{2} \hat{G}}{d y^{2}}+10 \lambda^{3} \frac{d^{4} \hat{G}}{d y^{4}}+3 \lambda \frac{d^{6} \hat{G}}{d y^{6}}\right) \\
F\left(H_{x y}\right)=-\frac{i}{R_{1}}\left(M K_{1}-R_{2}\right)\left[(L+2 M)\left(K_{1}-K_{2}\right)-2 R_{1}^{2}\right]\left(\lambda^{7} \hat{G}-3 \lambda^{5} \frac{d^{2} \hat{G}}{d y^{2}}+3 \lambda^{3} \frac{d^{4} \hat{G}}{d y^{4}}-\lambda \frac{d^{6} \hat{G}}{d y^{6}}\right) \\
-(L+M)\left(K_{1}-K_{2}\right) R_{1} \cdot\left(3 \lambda^{6} \frac{d \hat{G}}{d y}+10 \lambda^{4} \frac{d^{3} \hat{G}}{d y^{3}}+3 \lambda^{2} \frac{d^{5} \hat{G}}{d y^{5}}\right) \\
F\left(H_{y x}\right)=(L+M)\left(K_{1}-K_{2}\right) R_{1} \cdot i \cdot\left(3 \lambda^{5} \frac{d^{2} \hat{G}}{d y^{2}}+10 \lambda^{3} \frac{d^{4} \hat{G}}{d y^{4}}+3 \lambda \frac{d^{6} \hat{G}}{d y^{6}}\right) \\
F\left(H_{x y}\right)=(L+M)\left(K_{1}-K_{2}\right) R_{1} \cdot\left(3 \lambda^{6} \frac{d \hat{G}}{d y}+10 \lambda^{4} \frac{d^{3} \hat{G}}{d y^{3}}+3 \lambda^{2} \frac{d^{5} \hat{G}}{d y^{5}}\right)
\end{gathered}
$$

From Equation (21), we obtain the $n$ partial derivative of $\hat{G}$ as follows $(n=1,2, \cdots, 7)$,

$$
\begin{aligned}
& \frac{d \hat{G}}{d y}=e^{-|\lambda| y}\left(A_{2} \lambda-A_{1}|\lambda|\right)+y e^{-|\lambda| y}\left(2 A_{3} \lambda-A_{2} \lambda \cdot|\lambda|\right) \\
& +y^{2} e^{-|\lambda| y}\left(3 A_{4} \lambda-A_{3} \lambda \cdot|\lambda|\right)-A_{4} \lambda \cdot|\lambda| \cdot y^{3} e^{-|\lambda| y} \\
& \frac{d^{2} \hat{G}}{d y^{2}}=e^{-|\lambda| y}\left(A_{1} \lambda^{2}-2 A_{2} \lambda \cdot|\lambda|+2 A_{3} \lambda\right)+y e^{-|\lambda| y}\left(A_{2} \lambda^{3}-4 A_{3} \lambda \cdot|\lambda|+6 A_{4} \lambda\right) \\
& +y^{2} e^{-|\lambda| y}\left(A_{3} \lambda^{3}-6 A_{4} \lambda \cdot|\lambda|\right)+A_{4} \lambda^{3} \cdot y^{3} e^{-|\lambda| y} \\
& \frac{d^{3} \hat{G}}{d y^{3}}=e^{-|\lambda| y}\left(3 A_{2} \lambda^{3}-A_{1} \lambda^{2} \cdot|\lambda|-6 A_{3} \lambda \cdot|\lambda|+6 A_{4} \lambda\right)+y e^{-|\lambda| y}\left(-A_{2} \lambda^{3} \cdot|\lambda|+6 A_{3} \lambda^{3}-18 A_{4} \lambda \cdot|\lambda|\right) \\
& +y^{2} e^{-|\lambda| y}\left(-A_{3} \lambda^{3} \cdot|\lambda|+9 A_{4} \lambda^{3}\right)-A_{4} \lambda^{3} \cdot|\lambda| \cdot y^{3} e^{-|\lambda| y} \\
& \frac{d^{4} \hat{G}}{d y^{4}}=e^{-|\lambda| y}\left(-4 A_{2} \lambda^{3} \cdot|\lambda|+A_{1} \lambda^{4}+12 A_{3} \lambda^{3}-24 A_{4} \lambda \cdot|\lambda|\right)+y e^{-|\lambda| y}\left(A_{2} \lambda^{5}-8 A_{3} \lambda^{3} \cdot|\lambda|+36 A_{4} \lambda^{3}\right) \\
& +y^{2} e^{-|\lambda| y}\left(A_{3} \lambda^{5}-12 A_{4} \lambda^{3} \cdot|\lambda|\right)+A_{4} \lambda^{5} \cdot y^{3} e^{-|\lambda| y} \\
& \frac{d^{5} \hat{G}}{d y^{5}}=e^{-|\lambda| y}\left(5 A_{2} \lambda^{5}-A_{1} \lambda^{4} \cdot|\lambda|-20 A_{3} \lambda^{3} \cdot|\lambda|+60 A_{4} \lambda^{3}\right)+y e^{-|\lambda| y}\left(-A_{2} \lambda^{5} \cdot|\lambda|+10 A_{3} \lambda^{5}-60 A_{4} \lambda^{3} \cdot|\lambda|\right) \\
& +y^{2} e^{-|\lambda| y}\left(-A_{3} \lambda^{5} \cdot|\lambda|+15 A_{4} \lambda^{5}\right)-A_{4} \lambda^{5} \cdot|\lambda| \cdot y^{3} e^{-|\lambda| y} \\
& \frac{d^{6} \hat{G}}{d y^{6}}=e^{-|\lambda| y}\left(-6 A_{2} \lambda^{5} \cdot|\lambda|+A_{1} \lambda^{6}+30 A_{3} \lambda^{5}-120 A_{4} \lambda^{3} \cdot|\lambda|\right)+y e^{-|\lambda| y}\left(A_{2} \lambda^{7}-12 A_{3} \lambda^{5} \cdot|\lambda|+90 A_{4} \lambda^{5}\right) \\
& +y^{2} e^{-|\lambda| y}\left(A_{3} \lambda^{7}-18 A_{4} \lambda^{5} \cdot|\lambda|\right)+A_{4} \lambda^{7} \cdot y^{3} e^{-|\lambda| y} \\
& \frac{d^{7} \hat{G}}{d y^{7}}=e^{-|\lambda| y}\left(7 A_{2} \lambda^{7}-A_{1} \lambda^{6} \cdot|\lambda|-42 A_{3} \lambda^{5} \cdot|\lambda|+210 A_{4} \lambda^{5}\right)+y e^{-|\lambda| y}\left(-A_{2} \lambda^{7} \cdot|\lambda|+14 A_{3} \lambda^{7}-126 A_{4} \lambda^{5} \cdot|\lambda|\right) \\
& +y^{2} e^{-|\lambda| y}\left(-A_{3} \lambda^{7} \cdot|\lambda|+21 A_{4} \lambda^{7}\right)-A_{4} \lambda^{7} \cdot|\lambda| \cdot y^{3} e^{-|\lambda| y} \\
& \frac{d^{8} \hat{G}}{d y^{8}}=e^{-|\lambda| y}\left(-8 A_{2} \lambda^{7} \cdot|\lambda|+A_{1} \lambda^{8}+56 A_{3} \lambda^{7}-336 A_{4} \lambda^{5} \cdot|\lambda|\right)+y e^{-|\lambda| y}\left(A_{2} \lambda^{9}-16 A_{3} \lambda^{7} \cdot|\lambda|+168 A_{4} \lambda^{7}\right) \\
& +y^{2} e^{-|\lambda| y}\left(A_{3} \lambda^{9}-24 A_{4} \lambda^{7} \cdot|\lambda|\right)+A_{4} \lambda^{9} \cdot y^{3} e^{-|\lambda| y}
\end{aligned}
$$

Substitute Equation (2) into Equation (1) to get

$$
\begin{aligned}
F\left(\sigma_{x x}\right)= & D \cdot\left[e^{-|\lambda| y}\left(-8 A_{2} \lambda^{7}+64 A_{3} \lambda^{5} \cdot|\lambda|+108 A_{4} \lambda^{5}\right)+y \cdot e^{-|\lambda| y}\left(-16 A_{3} \lambda^{7}+192 A_{4} \lambda^{5} \cdot|\lambda|\right)\right. \\
& \left.+y^{2} \cdot e^{-|\lambda| y}\left(-24 A_{4} \lambda^{7}\right)\right] \\
F\left(\sigma_{y y}\right)= & D \cdot\left[e^{-|\lambda| y}\left(8 A_{2} \lambda^{7}-32 A_{3} \lambda^{5} \cdot|\lambda|+72 A_{4} \lambda^{5}\right)+y \cdot e^{-|\lambda| y}\left(16 A_{3} \lambda^{7}-96 A_{4} \lambda^{5} \cdot|\lambda|\right)\right. \\
& \left.+y^{2} \cdot e^{-|\lambda| y} \cdot 24 A_{4} \lambda^{7}\right] \\
F\left(\sigma_{x y}\right)= & \hat{\sigma}_{y x}=D \cdot i \cdot\left[e^{-|\lambda| y}\left(-8 A_{2} \lambda^{6} \cdot|\lambda|+48 A_{3} \lambda^{6}-168 A_{4} \lambda^{4} \cdot|\lambda|\right)+y \cdot e^{-|\lambda| y}\left(-16 A_{3} \lambda^{6} \cdot|\lambda|+144 A_{4} \lambda^{6}\right)\right. \\
& \left.+y^{2} \cdot e^{-|\lambda| y}\left(-24 A_{4} \lambda^{6} \cdot|\lambda|\right)\right]
\end{aligned}
$$




$$
\begin{aligned}
F\left(H_{x x}\right)= & -\frac{1}{R_{1}}\left(M K_{1}-R_{2}\right)\left[(L+2 M)\left(K_{1}-K_{2}\right)-2 R^{2}\right]\left(48 A_{4} \lambda^{5} \cdot e^{-|\lambda| y}\right) \\
& +(L+M)\left(K_{1}-K_{2}\right) \cdot R_{1} \cdot i \cdot\left[e^{-|\lambda| y} \cdot\left(16 A_{1} \lambda^{7}-64 A_{2} \lambda^{6} \cdot|\lambda|+216 A_{3} \lambda^{6}-600 A_{4} \lambda^{4} \cdot|\lambda|\right)\right. \\
& +y \cdot e^{-|\lambda| y}\left(16 A_{2} \lambda^{8}-128 A_{3} \lambda^{6} \cdot|\lambda|+648 A_{4} \lambda^{6}\right)+y^{2} \cdot e^{-|\lambda| y}\left(16 A_{3} \lambda^{8}-192 A_{4} \lambda^{6} \cdot|\lambda|\right) \\
& \left.+y^{3} \cdot e^{-|\lambda| y} \cdot 16 A_{4} \lambda^{8}\right] \\
F\left(H_{x y}\right)=- & -\frac{i}{R_{1}}\left(M K_{1}-R_{2}\right)\left[(L+2 M)\left(K_{1}-K_{2}\right)-2 R_{1}^{2}\right]\left[e^{-|\lambda| y} \cdot\left(-60 A_{4} \lambda^{4} \cdot|\lambda|\right)+y \cdot e^{-|\lambda| y}\left(A_{2} \lambda^{7} \cdot|\lambda|-A_{2} \lambda^{8}\right)\right. \\
& \left.+y^{2} \cdot e^{-|\lambda| y}\left(A_{3} \lambda^{7} \cdot|\lambda|-A_{3} \lambda^{8}\right)+y^{3} \cdot e^{-|\lambda| y}\left(A_{4} \lambda^{7} \cdot|\lambda|-A_{4} \lambda^{8}\right)\right] \\
& -(L+M)\left(K_{1}-K_{2}\right) \cdot R_{1} \cdot\left[e^{-|\lambda| y} \cdot\left(-16 A_{1} \lambda^{6} \cdot|\lambda|+48 A_{2} \lambda^{7}-120 A_{3} \lambda^{5} \cdot|\lambda|+240 A_{4} \lambda^{5}\right)\right. \\
+ & +y \cdot e^{-|\lambda| y}\left(-16 A_{2} \lambda^{7} \cdot|\lambda|+96 A_{3} \lambda^{7}-360 A_{4} \lambda^{5} \cdot|\lambda|\right)+y^{2} \cdot e^{-|\lambda| y}\left(-16 A_{3} \lambda^{7} \cdot|\lambda|+144 A_{4} \lambda^{7}\right) \\
+ & \left.y^{3} \cdot e^{-|\lambda| y} \cdot\left(-16 A_{4} \lambda^{7} \cdot|\lambda|\right)\right] \\
F\left(H_{y x}\right)= & (L+M)\left(K_{1}-K_{2}\right) \cdot R_{1} \cdot i \cdot\left[e^{-|\lambda| y} \cdot\left(16 A_{1} \lambda^{7}-64 A_{2} \lambda^{6} \cdot|\lambda|+216 A_{3} \lambda^{6}-360 A_{4} \lambda^{4} \cdot|\lambda|\right)\right. \\
& +y \cdot e^{-|\lambda| y}\left(16 A_{2} \lambda^{8}-128 A_{3} \lambda^{6} \cdot|\lambda|+648 A_{4} \lambda^{6}\right)+y^{2} \cdot e^{-|\lambda| y}\left(16 A_{3} \lambda^{8}-192 A_{4} \lambda^{6} \cdot|\lambda|\right) \\
& \left.+y^{3} \cdot e^{-|\lambda| y} \cdot 16 A_{4} \lambda^{8}\right] \\
F\left(H_{y y}\right)= & (L+M)\left(K_{1}-K_{2}\right) \cdot R_{1} \cdot\left[e^{-|\lambda| y} \cdot\left(-16 A_{1} \lambda^{6} \cdot|\lambda|+48 A_{2} \lambda^{7}-120 A_{3} \lambda^{5} \cdot|\lambda|+240 A_{4} \lambda^{5}\right)\right. \\
& +y \cdot e^{-|\lambda| y}\left(-16 A_{2} \lambda^{7} \cdot|\lambda|+96 A_{3} \lambda^{7}-360 A_{4} \lambda^{5} \cdot|\lambda|\right)+y^{2} \cdot e^{-|\lambda| y}\left(-16 A_{3} \lambda^{7} \cdot|\lambda|+144 A_{4} \lambda^{7}\right) \\
& \left.+y^{3} \cdot e^{-|\lambda| y} \cdot\left(-16 A_{4} \lambda^{7} \cdot|\lambda|\right)\right]
\end{aligned}
$$

Then $\mathcal{F}\left(\sigma_{i j}\right), \mathcal{F}\left(H_{i j}\right)$ can be obtained by substituting Equation (23) into Equation (3). By taking the Fourier inversion transform, the stresses $\sigma_{i j}, H_{i j}$ are determined.

\section{References}

1. Shechtman, D.; Blech, I.; Gratias, D.; Cahn, J.W. Metallic Phase with Long-Range Orientational Order and No Translational Symmetry. Phys. Rev. Lett. 1984, 53, 1951-1953. [CrossRef]

2. Landau, L.D.; Lifshitz, E.M. Statistical Physics; Pergamon Press: New York, NY, USA, 1958.

3. Bohsung, J.; Trebin, H.R. Disclinations in quasicrystals. Phys. Rev. Lett. 1987, 58, 1204-1207. [CrossRef] [PubMed]

4. Bak, P. Phenomenological theory of icosahedral in commensurate (quasiperiodic) order in $\mathrm{Mn}$-Al alloys. Phys. Rev. Lett. 1985, 54, 1517-1519. [CrossRef] [PubMed]

5. Socolar, J.E.S.; Lubensky, T.C.; Steinhardt, P.J. Phonons, phasons, and dislocations in quasicrystals. Phys. Rev. B 1986, 34, 3345-3360. [CrossRef] [PubMed]

6. Edagawa, K. Phonon-Phason coupling in decagonal quasicrystals. Philos. Mag. 2007, 87, 2789-2798. [CrossRef]

7. Chernikov, M.A.; Ott, H.R.; Bianchi, A.; Migliori, A.; Darling, T.W. Elastic Moduli of a Single Quasicrystal of Decagonal Al-Ni-Co: Evidence for Transverse Elastic Isotropy. Phys. Rev. Lett. 1998, 80, 321-324. [CrossRef]

8. Tanaka, K.; Mitara, Y.; Koiwa, M. Elastic constants of Al-based icosahedral quasicrystals. Philos. Mag. A 1996, 73, 1715-1723. [CrossRef]

9. Ding, D.-H.; Yang, W.; Hu, C.; Wang, R. Generalized elasticity theory of quasicrystals. Phys. Rev. B 1993, 48, 7003-7010. [CrossRef]

10. Hu, C.; Wang, R.; Ding, D.-H. Symmetry groups, physical property tensors, elasticity and dislocations in quasicrystals. Rep. Prog. Phys. 2000, 63, 1-39. [CrossRef]

11. Jeong, H.-C.; Steinhardt, P.J. Finite-Temperature elasticity phase transition in decagonal quasicrystals. Phys. Rev. B 1993, 48, 9394-9403. [CrossRef]

12. Levine, D.; Lubensky, T.C.; Östlund, S.; Ramaswamy, S.; Steinhardt, P.J.; Toner, J. Elasticity and Dislocations in Pentagonal and Icosahedral Quasicrystals. Phys. Rev. Lett. 1985, 54, 1520-1523. [CrossRef] [PubMed]

13. Coddens, G. On the problem of the relation between phason elasticity and phason dynamics in quasicrystals. Eur. Phys. J. B 2006, 54, 37-65. [CrossRef]

14. Fan, T.Y. Mathematical Theory of Elasticity of Quasicrystals and Its Applications; Springer: Berlin/Heidelberg, Germany, 2010.

15. Li, X.-F.; Duan, X.-Y.; Fan, T.-Y.; Sun, Y.-F. Elastic field for a straight dislocation in a decagonal quasicrystal. J. Phys. Condens. Matter 1999, 11, 703-711. [CrossRef]

16. Li, F.X.; Fan, T.Y.; Sun, Y.F. A decagonal quasicrystal with a Griffith crack. Philos. Mag. A 1999, 79, 1943-1952.

17. Li, L.H.; Fan, T.Y. Complex function method for solving notch problem of point 10 two-Dimensional quasicrystal based on the stress potential function. J. Phys. Condens. Matter 2006, 18, 10631-10641. 
18. Gao, Y.; Ricoeur, A. The Refined Theory of One-Dimensional Quasi-Crystals in Thick Plate Structures. J. Appl. Mech. 2011, 78, 031021. [CrossRef]

19. Wang, J.; Mancini, L.; Wang, R.; Gastaldi, J. Phonon and phason-type spherical inclusions in icosahedral quasicrystals. J. Phys. Condens. Matter 2003, 15, L363-L370. [CrossRef]

20. Radi, E.; Mariano, P.M. Stationary straight cracks in quasicrystals. Int. J. Fract. 2010, 166, 102-120. [CrossRef]

21. Radi, E.; Mariano, P.M. Steady-State propagation of dislocations in quasi-crystals. Proc. R. Soc. A Math. Phys. Eng. Sci. 2011, 467, 3490-3508. [CrossRef]

22. Mariano, P.M.; Planas, J. Phason self-actions in quasicrystals. Phys. D Nonlinear Phenom. 2013, $249,46-57$. [CrossRef]

23. Li, X.-Y. Fundamental solutions of penny-Shaped and half-Infinite plane cracks embedded in an infinite space of one-Dimensional hexagonal quasi-Crystal under thermal loading. Proc. R. Soc. A Math. Phys. Eng. Sci. 2013, 469. [CrossRef]

24. Li, X. Elastic field in an infinite medium of one-dimensional hexagonal quasicrystal with a planar crack. Int. J. Solids Struct. 2014, 51, 1442-1455. [CrossRef]

25. Li, W.; Xin, H.; Fan, T. Boundary Value Problems of Potential Functions in Decagonal Quasicrystals. Math. Probl. Eng. 2014, 2014, 1-8. [CrossRef]

26. Li, W.; Fan, T.Y. Dynamic Analysis of Cracked Octagonal Quasicrystals. Math. Probl. Eng. 2011, $2011,567489$. [CrossRef]

27. Wollgarten, M.; Beyss, M.; Urban, K.; Liebertz, H.; Koster, U. Direct evidence for plastic deformation of quasicrystals by means of a dislocationmechanism. Phys. Rev. Lett. 2003, 71, 549-552. [CrossRef] [PubMed]

28. Feuerbacher, M.; Bartsch, M.; Grushko, B.; Messerschmidt, U.; Urban, K. Plastic deformation of decagonal Al-Ni-Co quasicrystals. Philos. Mag. Lett. 1997, 76, 369-376. [CrossRef]

29. Messerschmidt, M.B.U. Friction mechanism of dislocation motion in icosahedral Al-Pd-Mn quasicrystals. Philos. Mag. A 1999, 79, 2123-2135. [CrossRef]

30. Schall, P.; Feuerbacher, M.; Bartsch, M.; Messerschmidt, U.; Urban, K. Dislocation density evolution upon plastic deformation of Al-Pd-Mn single quasicrystals. Philos. Mag. Lett. 1999, 79, 785-796. [CrossRef]

31. Geyer, B.; Bartsch, M.; Feuerbacher, M.; Urban, K.; Messerschmidt, U. Plastic deformation of icosahedral Al-Pd-Mn single quasicrystals I. Experimental results. Philos. Mag. A 2000, 80, 1151-1163. [CrossRef]

32. Rosenfeld, R.; Feuerbacher, M.; Baufeld, B.; Bartsch, M.; Wollgarten, M.; Hanke, G.; Beyss, M.; Messerschmidt, U.; Urban, K. Study of plastically deformed icosahedral Al[sbnd]Pd[sbnd]Mn single quasicrystals by transmission electron microscopy. Philos. Mag. Lett. 1995, 72, 375-384. [CrossRef]

33. Caillard, D.; Vanderschaeve, G.; Bresson, L.; Gratias, D. Transmission electron microscopy study of dislocations and extended defects in as-Grown icosahedral Al-Pd-Mn single grains. Philos. Mag. Lett. 2000, 80, 237-253. [CrossRef]

34. Muskhelishvili, N.I. Some Basic Problems of Mathematical Theory of Elasticity; P Noordhoff: Groringen, The Netherlands, 1956.

35. Mariano, P.M. Mechanics of Quasi-Periodic Alloys. J. Nonlinear Sci. 2005, 16, 45-77. [CrossRef]

36. Mariano, P.M.; Stazi, F.L.; Augusti, G. Phason effects around a crack in Al-Pb-Mn quasicrystals: Stochastic aspects of the phonon-phason coupling. Comput. Struct. 2004, 82, 971-983. [CrossRef]

(C) 2020 by the authors. Licensee MDPI, Basel, Switzerland. This article is an open access article distributed under the terms and conditions of the Creative Commons Attribution (CC BY) license (http://creativecommons.org/licenses/by/4.0/). 